$11-12-2020$

\title{
Kisumu, Kenya: Adult COVID-19 perceptions, prevention practices and impact-Responses from the first round of adult data collection in Kisumu, Kenya
}

Population Council

Follow this and additional works at: https://knowledgecommons.popcouncil.org/departments_sbsr-pgy How does access to this work benefit you? Let us know!

\section{Recommended Citation}

"Kisumu, Kenya: Adult COVID-19 perceptions, prevention practices and impact-Responses from the first round of adult data collection in Kisumu, Kenya," COVID-19 Research \& Evaluations brief. Nairobi:

Population Council, 2020. 


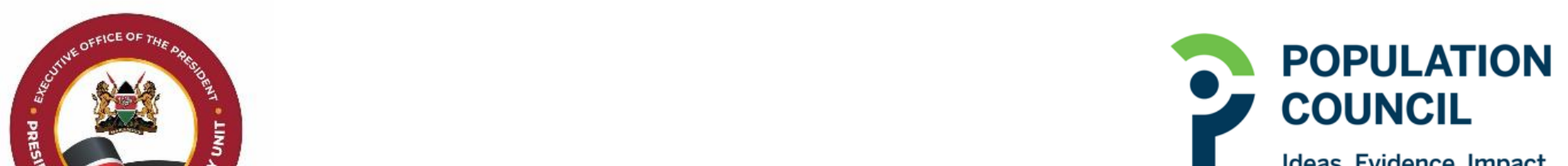

\title{
Kisumu, Kenya: Adult COVID-19 Perceptions, Prevention Practices and Impact
}

Responses from the first round of adult data collection in Kisumu, Kenya

\author{
August 2020
}

\section{H ighlights}

- Overall, knowledge of COVID-19 risk factors, symptoms and preventive behaviors in Kisumu are high. Perceived risk is mixed; those who perceive their risk as high mainly attribute this to interacting with a lot of people each day, higher for men (25\%) than women (16\%).

- Overall, compared to before COVID-19 almost all participants reported staying at home more (80\%), stoppingattending events (83\%), keeping a distance of 1-2 meters (90\%), washing hands more $(94 \%)$ and checking the news more $(78 \%)$.

- Fear of beingstigmatized if infected with COVID-19 is pervasive: $89 \%$ said if infected, people would gossip about them, $61 \%$ said people would treat their family badly, and after recovering, $68 \%$ people would still avoid them and $21 \%$ said they would not be welcomed back home -higher forfemales vs males (23\% vs $13 \%$ ).

- Almost half (47\%) of participants reported county health officials or community health workers had been working in their community to do education (87\%), install handwashingstations (75\%), and do contact tracing (34\%).

- The economic effects of the COVID-19 mitigation measures are significant: Over 4 out of 5 participants reported a complete or partial loss of income.

- Food insecurity is a major threat: $73 \%$ reported skipping a meal or eating less in the last week, more so women (75\%) than men (64\%).

- Despite large economic losses, assistance to adults in Kisumu is limited. Twenty percent of respondents reported receiving assistance for any reason from any institution.

\section{Knowledge, Attitudes, and Perceived Risk of Infection}

Between August $15^{\text {th }}-18^{\text {th }}$, a COVID-19 phone survey was completed with 858 adults in Kisumu, Kenya. The average age was 55 years, $70 \%$ were female and almost all own their own cell phone. Households were part the DREAMS program and evaluation cohort.. Kisumu county is a port city on Lake Victoria, densely populated compared to the rest of Kenya. As of August 2020, there were 66 confirmed cases of COVID-19 in Kisumu.

- Knowledge: Overall, respondents in Kisumu have high knowledge of COVID19 symptoms and transmission. Most knew that the elderly where at high risk if infected (71\%) and those with weakened immune systems (31\%), although half incorrectly stated children are at highest risk (54\%). Knowledge of fever (82\%), cough (79\%) and difficulty breathing (61\%) as symptoms was high.

- Prevention: Most adults (95\%) correctly identified handwashing as a method to prevent the spread of COVID-19 and over three-quarters

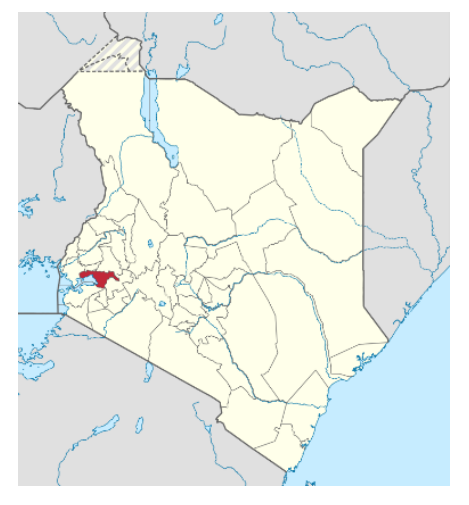


identified wearing masks ( $83 \%$ ) and standing 1 to 2 meters apart ( $80 \%$ )as prevention methods.

- Information source: Government channels were the most reported sources of COVID-19 information: Government radio advertisements (90\%), Government televisionadvertisements (83\%), and government SMS (78\%). Almost everyonesaid these were trustworthy sources of information. Overall, $40 \%$ used social media for information; this was higher for high education respondents ( $71 \%$ of those with higher education compared to $21 \%$ of those with primary school or less) and highest for younger respondents ( $61 \%$ of $20-24$ year olds vs $19 \%$ of $45+$ year olds).

- Stigma: concerns regarding stigma were extremely high, about three-quarters reported that if infected, people would stop talking to them (73\%), people would stop visit their house/business (84\%), and people would gossip about them (89\%). Only half said people they know would bring them food or medicine if infected (51\%). After recovering from COVID-19, abouta quarter of women (23\%) said they would not be welcome back into their own home, compared to $13 \%$ of men.

- Experience with COVID-19: Few respondents (4\%) reported knowing someone who has tested positive for COVID-19 or was suspected of being positive, higher for men (7\%) than women (3\%) but overall quite low. $8 \%$ reported being tested themselves.

- Perceived risk: Overall, over a quarter of adults (28\%) consider themselves to be at high risk of COVID-19 infection. Among those who have low perceived risk (32\%), the main reasons are: staying home (23\%) and carrying out recommended prevention behaviors, including social distancing (39\%), wearing a mask (35\%), and washing hands (39\%). Few responded their low risk was because they are young $(2 \%)$ or god protects me $(2 \%)$.

\section{COVID-19 Prevention M ethods}

\section{So cial Distancing / Wearing Face Masks}

- Both men and women reported interacting with others less than before the COVID-19 pandemic began. Overall, $65 \%$ are avoiding public transportation more, $67 \%$ are seeing friends less, and $45 \%$ are seeing family less.

- Almost all respondents (98\%) worea face mask in the last week; 93\% said always.

\section{Handwashing}

- Almost all (99\%) of respondents report that there are handwashing stations in public areas near their home, and $93 \%$ have a designated place in their home to wash hands. All respondents have water (100\%) and soap (99\%) for handwashing.

- $67 \%$ say they wash hands after using the toilet and $69 \%$ before eating, which were the common pre-COVID-19 recommendations for handwashing. Many respondents also wash their hands after coming home from a public place (78\%) but few did after caring for sick people (4\%).

\section{Awareness of government intervention}

- Almost half (47\%) of participants reported seeing county health officials or community health workers working in their community to do education (87\%), install handwashing stations (75\%), and do contact tracing (34\%).

\section{Impact of the COVID-19 M itigation Response}

The COVID-19 pandemic and associated social distancing and lockdown measures have had a large economic, health, and social impact on respondents. Many are earning less income due to COVID-19 even as costs of household items have risen, and a substantial percentage report experiencing food insecurity. Relative to need, very few adults are receiving assistance in the form of cash, vouchers, or essential items. 
- A quarter (26\%) of participants reported complete loss of income, and $57 \%$ reporteda partial loss of income. Women were more likely to report complete loss while men were more likely to report a partial loss; overall, any loss of income was higher among women.

- Overall, $85 \%$ of respondents report earning less in the past month than they earned before the pandemic began, and $58 \%$ say their partner is also earning less. Meanwhile, participants reported increasing costs of food (89\%), cooking fuel (72\%) and overall increased expenses for the household (79\%).

- Women are taking on more unpaid labor: they are more likely than men to report they are doing more cooking ( $55 \%$ vs. $24 \%$ ), fetching water ( $50 \%$ vs $17 \%$ ), cleaning ( $69 \%$ vs. $19 \%$ ), and childcare (64\% vs. $24 \%$ ) since the pandemic began.

\section{Food Security}

- Many participants have been skipping meals or eating less since the COVID-19 pandemic began. In the past 7 days, $75 \%$ of women and $64 \%$ of men skippeda meal, with $95 \%$ of those individuals reporting that the skipped meal was due to COVID-19 disruptions.

- $69 \%$ say they skipped a meal on a couple days last week and $11 \%$ say they skipped every day.

- When asked what their biggest need is that's not being addressed, $51 \%$ of respondents said cash, followed by food (41\%); men mentioned cash more and women mentioned foo $d$ more.

\section{Health and Safety}

- Access to health services is relatively high, $57 \%$ had been to a health facility since the start of COVID-19, and 91\% received the care they were seeking. About a quarter (24\%) said the service or commodity they sought was not available.

- All participants said providers were wearing masks (99\%) and that there was a handwashing station at the facility (100\%).

- Only $9 \%$ of participants reportedforgoing health services, the main reason being cannot afford it right now (36\%) followed by scared of getting COVID-19 at the clinic (17\%).

- In the past one week, $48 \%$ of participants reported feeling little interest or pleasure in the things they normally do and $50 \%$ reported feeling down, depressed, or hopeless. More than half say this is more so than before COVID-19.

- Participants reported increase crime in the neighborhood (45\%) and violence outside the home (25\%) since the start of COVID-19. Respondents also report that violence or tensions have increased within their households since the COVID-19 pandemic began ( $8 \%$ and $41 \%$ respectively).

\section{Conclusions and Recommendations}

COVID-19 public education campaigns in Kisumu should continue with a focus on:

- Addressing stigma and ensuring that those who test positive for COVID-19 receive needed food and medical care.

- Promote handwashing or sanitizer use after returning home and after caring for a sick household member.

Address the health, economic, and social impacts of lockdowns:

- Government and NGO assistance to adults in Kisumu has been minimal despite large numbers forgoing meals and experiencing com plete or partial income loss. Assistance in the form of food, vouchers, or cash should be provided and targeted to those with the greatest need.

- Resources to address the high increases in violence and tension, both in the community and within households are needed. 
The Population Council conducts research and delivers solutions to improve lives. As COVID-19 spreads around the world, the Population Council is working to lessen the health, social, and economic impact of the outbreak. On the ground in 50 countries and 14 offices, our experts are partnering with governments to develop approaches for rapid research, data collection, monitoring, and mapping, and to test and evaluate behavioral interventions.

Suggested citation: Population Council. 2020. “Kisumu, Kenya: Adult COVID-19 Perceptions, Prevention Practices, and Impact. Responses from the first round of adult data collection in Kisumu, Kenya"

(c) 2020 The Population Council, Inc.

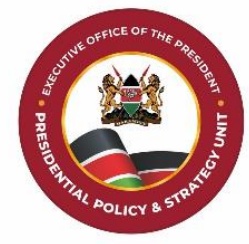

POPULATION COUNCIL

Ideas. Evidence. Impact. 